\title{
Tangence
}

\section{Jeux bibliques d'intertextualité ou l'impossibilité de lire hors de la bibliothèque}

\section{Jean-Jacques Lavoie}

Numéro 35, mars 1992

Des écritures à lire

URI : https://id.erudit.org/iderudit/025698ar

DOI : https://doi.org/10.7202/025698ar

Aller au sommaire du numéro

Éditeur(s)

Tangence

ISSN

0226-9554 (imprimé)

1710-0305 (numérique)

Découvrir la revue

Citer cet article

Lavoie, J.-J. (1992). Jeux bibliques d'intertextualité ou l'impossibilité de lire hors de la bibliothèque. Tangence, (35), 46-58. https://doi.org/10.7202/025698ar d'utilisation que vous pouvez consulter en ligne.

https://apropos.erudit.org/fr/usagers/politique-dutilisation/ 


\title{
Jeux bibliques d'intertextualité ou l'impossibilité de lire hors de la bibliothèque \\ Jean-Jacques Lavoie
}

\begin{abstract}
Plus ou moins tous les livres contiennent la fusion de quelque redite comptée.
\end{abstract}

Stéphane Mallarmé

\section{Prélude}

Depuis déjà plusieurs années, l'intertextualité a fait l'objet de nombreuses et savantes études ${ }^{1}$. Face à un tel foisonnement qui témoigne de l'intérêt que les chercheurs ont porté au concept, notre projet ne vise aucunement à l'exhaustivité. Un tel objectif serait d'ailleurs illusoire puisque le terme d'intertextualité est devenu l'objet d'une réflexion extrêmement complexe et polémique. On comprendra également que l'objectif de cet article n'est pas d'ajouter une théorie supplémentaire de l'intertextualité à la longue liste déjà existante; les problèmes sont déjà assez nombreux dans ce domaine pour ne pas en ajouter un de plus. Néanmoins, nous nous interrogerons sur le sens que nous donnons à ce mot en prenant soin de signaler quelques points de repères dans la recherche contemporaine.

Une fois que nous aurons reconnu qu'il n'y a pas d'énoncé qui soit dépourvu de dimension intertextuelle, nous examinerons

1 On trouvera une bibliographie qui comprend 339 titres dans D. Bruce, - Bibliographie annotée. Ecrits sur l'intertextualité ., Texte, 2 (1983) p. 217 258. Par ailleurs, du point de vue biblique, la recherche est quasi inexistante. On peut noter un numéro spécial de Sémiotique et Bible, 15 (1979), qui consacre quelques réflexions aux problèmes de l'intertextualité et un collectif édité par S. Draisrna, Intertextuality in Biblical Writings. Essays in Honour of Bas Van lersel, Kampen, Uitgevers-maatschappij J. H. Kok, 1989, qui se voue presque entièrement à des analyses de textes néotestamentaires. En outre, on peut signaler notre livre intitulé $A u$ sortir de l'Éden: ou comment ne plus tricher? L'absurde dans l'œuvre du Qobélet, Montréal, Fides (à paraître), qui aborde le Qohélet sous l'angle de l'intertextualité. 
les notions de citation et d'allusion en tant que modes d'insertion de l'intertextualité. Cette deuxième partie sera déjà un peu plus originale que la première. En effet, malgré les nombreuses monographies sur ce sujet, peu de méthodes ont été proposées pour conduire les études intertextuelles.

Enfin, comme ces mots ne trouvent leur légitimation que dans l'étude de la pratique, dans leur insertion à l'intérieur de textes précis, nous donnerons quelques exemples. Ceux-ci proviendront exclusivement de la Bible.

\section{A la recherche d'une définition}

Comme signifiant, le mot *intertexte * est ancien. Il existait déjà en latin où intertextus était le participe passé passif du verbe intertexere qui signifiait *entrelacer ", "entremêler *, "assembler *, "combiner. L'idée d'une relation (inter) entre des énoncés (textes) qui constituent l'intertexte est aussi déjà présente dans les recherches de M. Bakhtine. Il avait toutefois baptisé de * dialogisme * cette relation complexe de chaque énoncé aux autres énoncés ${ }^{2}$. C'est à une lectrice de Mikhail Bakhtine, Julia Kristeva, que nous devons la notion d'intertextualité. Le mot revient pour les premières fois dans plusieurs de ses essais écrits en 1966-1969, parus dans Critique, Tel Quel et Sémetotikè. Par exemple, en 1968, elle voyait dans l'intertextualité une *interaction textuelle qui se produit à l'intérieur d'un seul texte ${ }^{3}$. C'est en 1976 que sa définition se complexifie: l'intertextualité désigne alors *le croisement de la modification réciproque des unités appartenant à des différents textes "4. À partir de ce moment, l'originalité de Julia Kristeva fut de penser l'intertextualité non seulement à partir de la simple notion de relation, mais aussi et surtout à partir de la nature transformationnelle de cette relation.

Depuis les travaux de Kristeva, les théoriciens n'en finissent plus d'expliciter le sens de l'intertextualité. On parle de co-habitation, de co-présence, d'univers relationnel, d'univers d'alliances,

2 À ce propos, voir Tzvetan Todorov, Mikhaill Bakbtine. Le principe dialogique suivi des Ecrits du cercle de Bakbtine, Paris, Seuil, 1981, particulièrement les pages 95-115.

3 Julia Kristeva, "Problemes de la structuration du texte ", dans Théorie d'ensemble, Paris, Seuil, 1968, p. 297-316, p. 311.

4 Le texte du roman, approcbe sémiologique d'une structure discursive transformationnelle, Paris, De Gruyter, 1976, p. 68. 
mais aussi de travail d'assimilation, de transformation, de travail de ré-écriture, de re-création, etc. Mais aucune de ces nouvelles explications ne paraît avoir véritablement enrichi les définitions proposées par J. Kristeva ${ }^{5}$. Ce trop bref aperçu laisse pourtant soupçonner, en dépit des nombreuses définitions plus ou moins synonymiques, que l'intertextualité se prête à plusieurs approches théoriques dont les perspectives sont tantôt abstraites et sémiologiques, tantôt concrètes et fonctionnelles. Mais comme ces multiples approches ne trouvent leur légitimation que dans l'étude de la pratique, dans notre cas la Bible, nous préférons nous en tenir à la définition simple et claire de J. Kristeva, quitte à préciser certaines de nos affirmations au cours des pages qui vont suivre.

\section{Deux modes d'insertion de l'intertextualité: la citation et l'allusion}

Définir l'intertextualité c'est aussi la cerner dans ses modes d'insertion. Il faut donc nous poser la question suivante: quels sont les aspects majeurs et les modes d'insertion de l'intertextualité? Pour notre propos, nous n'en retiendrons que deux: la citation et l'allusion.

L'absence d'un critère sûr pour établir la distinction entre citation et allusion se vérifie facilement par l'écart de calcul établi par les spécialistes du Nouveau Testament. Ainsi le nombre des citations du Nouveau Testament est estimé par certains à environ 150 et par d'autres à 300, voire même à 400 et plus. La même confusion règne chez les spécialistes de l'Ancien Testament. Mais il serait naif de croire que cette imprécision est le seul lot des exégètes de la Bible. C'est ce que nous prouve clairement le très beau livre d'Antoine Compagnon qui porte précisément sur la citation et qui affirme d'emblée qu'on ne peut parler de la citation pour elle-même comme les critiques littéraires tentent de le faire, en donnant sans cesse des définitions qui s'opposent les unes aux autres. Par contre, on peut parler de son travail:

5 Cependant, cette affirmation ne doit pas laisser croire qu'il y a unanimité chez tous les théoriciens de l'intertextualité. Au contraire! Pour s'en convaincre, on trouvera une présentation de quelques problèmes dans Michel Arrivé, Intertexte et intertextualité chez Ferdinand de Saussure? :, Le plaisir de l'intertexte: formes et fonctions de l'intertextualité, Berne, Verlag Petersburg, 1986, p. 11-31, p. 14-15. 
Lire ou écrire, c'est faire acte de citation. La citation represente la pratique première du texte au fondement de la lecture et de l'écriture [...] Toute pratique du texte est toujours citation et c'est pourquoi, de la citation, aucune définition n'est possible. ${ }^{6}$

Il est plutôt remarquable qu'un tel aveu introduise un livre de 416 pages sur la citation! "La citation, poursuit-il à la page 38, n'a pas de sens en soi, parce qu'elle n'est que dans un travail, qui la déplace et qui la fait jouer. * Toutefois, dans sa forme la plus simple et la plus superficielle, Antoine Compagnon envisage la citation comme la "répétition d'une unité de discours dans un autre discours * (p. 54). Mais c'est à la page 56 que se précise sa définition:

Une *bonne " définition de la citation, c'est-à-dire une base acceptable, provisoire de travail, sera: un énoncé répété et une énonciation répétante; il ne faut jamais cesser de l'envisager dans cette ambivalence, la collusion, la confusion en elle de l'actif et du passif.

L'intérêt portée à l'énonciation montre très bien qu'il situe la citation non pas du côté du produit, mais bien du côté de l'acte. Nous partageons donc, d'une certaine façon, le soupçon qui est à la base des recherches sur l'intertextualité: et si l'écriture (avec ou sans majuscule) n'était en très grande partie qu'un tissu de citations, pour employer une expression devenue populaire de Roland Barthes, un tissu tissé entièrement avec des références et des échos à des langages culturels antérieurs ou contemporains, même là où il n'y a aucune trace évidente d'altérité. Tout texte ne contient-il pas, à des degrés différents, une dimension de citations? Par exemple, le seul fait que les mots d'un texte viennent d'ailleurs, qu'ils évoquent les discours qui les ont déjà portés et travaillés sont autant de perspectives de citations. Ainsi, la Bible, comme toute écriture, a donc un quelconque statut d'altérité et c'est ce statut d'altérité, cette tension du même et de l'autre, qui constitue, en grande partie, sa lisibilité. Autrement dit, si toute textualité est en quelque sorte intertextualité, un texte biblique, d'une certaine manière, vaut ce que vaut son action intégratrice et destructrice des autres textes. Le jeu de la citation s'effectue donc dans la négation et le maintien simultanés de la différence. Ce paradoxe du même et de l'autre se joue dans l'intégration de l'énoncé cité dans le discours porteur. En d'autres mots, la nouveauté n'est pas tant dans la

6 Antoine Compagnon, La seconde main ou le travail de la citation, Paris, Seuil, 1979, p. 34 . 
répétition que dans la position et la fonction que l'énoncé répété vient occuper dans le nouvel ensemble textuel.

Quant à l'intertextualité biblique, il est évident qu'elle se présentera presque toujours comme une citation indirecte et implicite qui ne comporte pas de renvoi à une référence précise. Peut-être devrions-nous croire qu'il y a contradiction dans les termes à imaginer une citation implicite, mais il ne saurait en être autrement pour les textes bibliques puisque les scribes de l'Antiquité ignoraient les caractères d'imprimerie comme les guillemets ou l'italique. Peut-être devrions-nous preférer parler de plagiat? Après tout, la citation n'est-elle pas un plagiat civilisé? Le plagiat n'est-il pas une citation inavouée? Mais une telle terminologie est inapplicable à la Bible et à son univers, car elle suppose la notion de propriété littéraire, laquelle est inévitablement liée à la notion d'auteur. Or, d'une part, la notion de droit d'auteur est une question littéraire et une loi pénale très récente dans l'histoire (fin du XVIII' et début du XIX $^{c}$ siècle) et, d'autre part, la Bible n'est pas un livre d'auteurs, mais une littérature de traditions. Par contre, la Bible n'ignore pas totalement les citations explicites, c'est-à-dire des énoncés précédés d'une formule d'introduction?. Il est important ici de préciser que le caractère explicite d'une citation biblique ne saurait être compris autrement. Un exemple suffira à éclairer notre propos. Le texte de 2 Cor 6,16b-18 comprend une formule d'introduction, mais cite neuf textes sans aucun scrupule d'exactitude. Par contre, Mc 4,12 n'a aucune formule d'introduction et pourtant le texte du Targum d'Is 6,9-10 est cité presque mot à mot.

Notre deuxième forme d'intertextualité est constituée par l'allusion. Étant encore plus implicite et moins littérale que notre premier mode, l'allusion exige donc un effort de décodage de la part du lecteur. On sait que jusqu'au XVII' siècle l'allusion désignait un jeu de mots, le latin allusio venant de ludere. Aujourd'hui, l'allusion est un énoncé dont la compréhension suppose la perception d'un rapport entre un texte présent et un texte absent, et dont ce rapport même réveille l'idée.

7 Par exemple, dans le cas du Nouveau Testament, l'addition de ces formules d'introduction élève le nombre de citations explicites à environ 140. C'est le chiffre qu'on obtient si l'on ne prend en considération que les citations marquées d'un astérisque dans la liste de L. Venard, - Citations de l'Ancien Testament dans le Nouveau,, Supplément au Dictionnaire de la Bible, 2 (1934), p. 24-28. 
Concrètement, le problème que pose l'allusion est donc le même que pour la citation: il s'agit de savoir si le lecteur voit ou ne voit pas le texte en filigrane qui a été métamorphosé, transposé et assimilé. C'est donc une fois de plus la sagacité du lecteur qui est en question.

Par conséquent, notre étude sur l'intertextualité biblique n'aura pas pour but de savoir si tel ou tel auteur avait bien tel ou tel texte sous les yeux pour composer son cuvre. Il faut être conscient du problème épistémologique que pose l'interprétation du texte biblique, de tout texte, qui sépare inexorablement l'auteur du lecteur et même l'auteur de sa propre intentionnalité. Le sens ne se trouve guère dans le texte biblique comme un dépôt qui gît paralysé et embaumé à travers les siècles. Lire la Bible ce n'est pas affronter un mort, mais réveiller les dires qui sommeillent dans.le dit et qui ne demandent qu'à naître. C'est donc dire que le sens se joue dans le rapport entre le texte et son lecteur. En d'autres mots, lire la Bible c'est jouer à la Bible, jouer la Bible; jouer, ici, étant aussi un terme musical. Cette considération de la lecture biblique comme l'exécution d'une partition musicale marque bien *l'effectuation, la venue à l'acte, des possibilités sémantiques du texte.8. Par le fait même, comme partition musicale, le texte biblique est susceptible d'exécutions différentes. C'est ce qu'illustre à merveille l'intertextualité biblique et c'est ce que nous allons maintenant examiner.

\section{Intertextualité dans l'Ancien Testament}

L'Ancien Testament est en grande partie un tissu de citations et d'allusions ${ }^{9}$. Par exemple, dans le Pentateuque, Dt 4,16-19a se présente au lecteur perspicace comme une série d'allusions à $\mathrm{Gn}$ 1,14-2,1:

8 P. Ricceur, Du texte à l'action. Essais d'berméneutique II, Paris, Seuil, 1986, p. 153. Autrement dit, c'est la lecture qui manifeste l'inachèvement du texte.

9 À propos de l'intertextualité et de ses modes d'insertion, on trouvera de nombreux exemples de - relectures bibliques. dans le livre de M. Fishbane, Biblical Interpretation in Ancient Israël, Oxford, Clarendon Press, 1985, et dans le collectif D.A. Carson et al., It is Written: Scripture Citing Scripture. Essays in Honour of Barnabas Lindars, Cambridge University Press, Cambridge, 1988. Les exemples qui vont suivre sont parfois inspirés de ces recherches, mais notre analyse des textes bibliques se démarque pour une bonne part de ces études puisque notre approche méthodologique est complètement différente. 
N'allez pas vous corrompre en vous fabriquant une idole, une forme quelconque de divinité, l'image d'un bomme ou d'une femme [cf. Gn 1,26a.27 bien que le mot pour dire *image * soit different], l'image de n'importe quelle bête de la terre [cf. Gn 1,24-25] ou de n'importe quel oiseau qui vole dans le ciel [cf. Gn 1,20b.21b.22bl, l'image de n'importe quel bestiole qui rampe sur le sol [cf. Gn 1,24-26], ou de n'importe quel poisson [cf. Gn $1,26.28$ ] qui vit dans les eaux sous la terre [cf. Gn 1,20a.21a.22a]. $\mathrm{Ne}$ va pas lever les yeux vers le ciel, regarder le soleil, la lune et les étoiles [cf. Gn 1,14-18a], toute l'armée des cieux [cf. Gn 2,1], et te laisser entraîner à te prosterner devant eux et à les servir.

La théologie de la création est ici subtilement associée à une éthique théologique pour montrer que l'idolâtrie est un péché contre le Créateur. Le premier récit de création devient plus précisément le lieu herméneutique où se joue la polémique deutéronomique contre la fabrication des idoles.

La littérature prophétique offre aussi plusieurs exemples d'intertextualité biblique. Ainsi, pour parler de la corruption morale de Juda et du châtiment divin qu'il mérite, Jérémie, le prophète de l'exil, n'hésite pas à reprendre les anciennes accusations du prophète Osée (12,1-15) contre Israël ${ }^{10}$ en citant les mêmes mots clés de l'histoire des frères Jacob et Esaü en Gn 25ss:

Gardez-vous chacun de son ami et que nul ne se fie à son frère [le mot revient 40 fois dans Gn 25,19-35,29]; car tout frère ne fait que supplanter [cf. Os 12,4 et $\mathrm{Gn} 27,36]$ et tout ami s'en va en calomniant. Chacun se joue [cf. Gn 31,7] de son ami et ils ne disent pas la vérité, ils ont instruit leur langue à dire le mensonge; ils font le mal, incapables de se convertir. Violence sur violence! Tromperie sur tromperie (cf. Os 12,8 et Gn 27,35; 34,13)! (Jr 9,3-5)

Ez 18,1-3 nous présente une citation où le texte porteur se fait très polémique:

La parole de Yahvé m'advint en ces termes: Qu'avez-vous à proférer ce proverbe en terre d'Israël: * Les pères ont mangé du raisin vert et les dents des fils ont été agacées"? Par ma vie! oracle du Seigneur Yahvé - vous n'aurez plus à proférer ce proverbe en Israël.

10 Lequel chapitre 12 est lui-même un tissu de citations et d'allusions à $\mathrm{Gn}$ 25ss: cf. Os 12,4a et Gn 25,26; Os 12,4b-5a et Gn 32,23-33; Os 12,5a et Gn 32,27 ; Os 12,5 b et Gn 28,$13 ; 35,15$; Os 12,7 et Gn $28,15.21 ; 31,13$; Os 12,9 et Gn $30,2831,10$; Os $12,13 a$ et Gn $27,43 s s ; 28,5 ; 29,1$ ss; Os $12,13 b$ et Gn $29,15-30$. 
La citation, comme l'indiquent les guillemets que nous avons pris soin de mettre, se trouve dans le proverbe populaire qui revient aussi en Jr 31,29. Bien sûr, il s'agit ici d'une citation de la tradition orale et celle-ci restera à jamais invérifiable. Néanmoins, le contexte indique clairement qu'il s'agit bien d'une citation. D'ailleurs, tout proverbe n'est-il pas citation? En effet, les proverbes sont presque toujours populaires, du moins à l'origine. Ils viennent du peuple, ils appartiennent au peuple. C'est pourquoi on peut dire des proverbes qu'ils sont essentiellement du *déjà-dit *, du "déjà-lu *. Autrement dit, toute pratique proverbiale dans un texte témoigne toujours de l'altérité. Or, ce proverbe, ce micro-récit a ici un signifié littéral et un signifié contextuel. Son signifié littéral est clair: il s'agit d'une expression imagée de la doctrine traditionnelle de la rétribution qui affirme que l'individu est responsable pour son clan, dans le présent comme dans l'avenir ( $c f$. Dt 24,16; Ex 20,$5 ; 34,7 ;$ etc.). Toutefois, le véritable enjeu réside dans l'interprétation du texte porteur et dans l'intégration du texte porté. Or, le commentaire du texte porteur est également évident: cette conception de la solidarité humaine n'a plus aucune raison d'être. Puis, cette négation est explicitée au verset suivant qui peut se lire comme une citation de Dt 24,16:

Par cette deuxième citation, Ezéchiel s'assure donc de miner cette vieille doctrine de la responsabilité collective. Bref, cette lecture intertextuelle nous dévoile deux citations de dérision.

Voici: toutes les personnes sont à moi; aussi bien la personne du fils que la personne du père, elles sont à moi: la personne qui pèche Imais le mot est différentl c'est elle qui mourra. (Ez 18,4)
Les pères ne seront pas mis à mort pour les fils, et les fils ne seront pas mis à mort pour les pères; c'est à cause de son péché que chacun sera mis à mort. (Dt 24,16)

Dans l'Ancien Testament, la littérature sapientiale reste le lieu privilégié pour une approche intertextuelle, puisque son herméneutique didactique se caractérise essentiellement par son remploi systématique des écrits antérieurs. C'est ce que G.T. Sheppard a appelé la "sapientialisation de l'Ancien Testament "11.

À lui seul, le livre de Job nous offre plusieurs exemples d'intertextualité biblique. Par exemple, Jb 7,17-19 est une citation

11 Wisdom as a Hermeneutical Construct. A Study in the Sapientializing of the Old Testament, Berlin-New York, Walter de Gruyter, 1980, 178 p. 
54

parodique, au sens étymologique même du terme, du Ps 8 qui est lui même un commentaire de Gn 1:

Qu'est-ce qu'un mortel, pour que tu t'en souviennes et un fils d'homme, pour que tu le visites? Tu l'as fait de peu inferieur à un dieu, de gloire et de splendeur tu l'as couronné; tu l'as fait dominer sur l'œuvre de tes mains, tu as tout mis sous ses pieds. (Ps, 8,5-7)
Qu'est-ce qu'un mortel pour en faire un tel cas, pour mettre sur lui ton attention, le visiter chaque matin, le scruter à tout instant? Quand cesseras-tu de me regarder? Ne me laisseras-tu pas le temps d'avaler ma salive? (Jb 7,17-19)

Tandis que le psaume exprime à la fois l'infinie majesté de Dieu $(8,1-5)$ et la dignité et le pouvoir que l'homme a reçu de son Créateur $(8,6-9)$, la question de Job au verset 17 , d'une part, n'exprime plus que l'insignifiance humaine $(c f .7,16)$ et, d'autre part, elle se trouve insérée dans un contexte qui accuse Dieu d'être insupportable. Comme l'exige la logique parodique, Jb 7,16-19 n'est donc qu'une citation détournée de son sens et de son contexte. Bien sûr, on pourrait interpréter ce passage sans faire appel au Ps 8, mais il n'en demeure pas moins que ce passage gagne en profondeur lorsqu'il est lu comme un lieu où se joue une intertextualité comme détournement de sens. C'est d'ailleurs ce que fera également Éliphaz, dans sa réplique, avec une citation tout aussi conflictuelle:

Qu'est-ce qu'un mortel pour qu'il soit pur, et pour que soit juste un enfant de la femme? À ses saints même il ne se fie pas, et le ciel n'est pas pur à ses yeux $(9,14-15)$

Éliphaz reprend ici la parodie de Job, comme l'indique la question en $14 \mathrm{a}$, mais pour lui rappeler, d'une part la transcendance de Dieu et, d'autre part, à partir d'un jeu de mots entre "pour qu'il soit pur. (yizkeb) et l'expression du Ps 8,5 *pour que tu t'en souviennes. (tizkerennû), la finitude humaine. Ces deux exemples montrent bien que lire un texte, c'est toujours lire le texte-là (identité) et son autre (différence); c'est toujours en lire plusieurs qui se cachent plus ou moins bien.

Le Cantique des Cantiques est lui aussi un texte promis à des embrassements, à des étreintes. Bien sûr, dans les limites de ce bref article on ne peut pas faire la démonstration que les allusions à Gn 1-3 sont la clé herméneutique du Cantique. Néanmoins, un seul texte sera suffisant pour illustrer de façon 
suggestive notre affirmation. Il s'agit d'une allusion à Gn 3,16 qu'on retrouve en $\mathrm{Ct} 7,11$ :

Je suis à mon bien-aimé, et vers moi est son désir. (Ct 7,11)
Et à la femme il dit: : Je multiplierai ta peine et tes grossesses, c'est dans la peine que tu enfanteras des fils, vers ton mari se portera ton désir et lui dominera sur toi. * (Gn 3,16)

L'allusion repose certes sur un seul mot, mais ce mot (shâ) ne revient qu'une seule autre fois dans la Bible: en Gn 4,7, verset situé à l'intérieur d'une péricope qui est une reprise de Gn 3,9ss. Par la reprise de ce mot rare, Ct 7,11 est donc un véritable détournement de texte à transformation maximale. La malédiction de Gn 3,16 est changée en bénédiction. Le désir est une joie et non un jugement. La relation amoureuse n'est plus unilatérale, mais réciproque $(\mathrm{Ct} 7,11 \mathrm{a})$. En outre, ce n'est plus la femme qui soupire auprès de l'homme comme en Gn 3,16, mais l'homme auprès de la femme. Ici peut-être plus qu'ailleurs, les mots font l'amour, selon la belle expression de Breton.

\section{Intertextualité dans le Nouveau Testament}

Avec ses nombreuses citations et allusions scripturaires, le Nouveau Testament est le lieu où se joue l'Autre Testament, d'emblée pluriel. Ces citations et allusions sont si nombreuses qu'on peut affirmer que le Nouveau Testament ne se donne presque jamais à lire au premier degré. Lire le Nouveau Testament, c'est presque toujours lire un autre texte à travers celui qui semble offert. Autrement dit, le Nouveau Testament nous convoque essentiellement à une lecture palimpsestueuse. Des exemples nous montreraient bien que les fonctions des citations et des allusions dans le Nouveau Testament répondent, en gros, à des préoccupations de deux ordres: d'une part, l'apologétique et la controverse; d'autre part, la théologie et la pastorale. Par exemple, les citations apologétiques de l'Ancien Testament dans les Actes varient, selon les exégètes, entre 18 et 50 ( $c f$. par exemple Ac 1,20 et Ps 69,$26 ; 2,17-21$ et Jl 3,1-5; 2,25-28 et Ps 16,8$11 ; 2,34-35$ et Ps 110,$1 ; 3,22-23$ et Dt 18,15-19; 3,25 et Gn 12,3; etc.). Bien sûr, cette distinction entre controverse et théologie est bien relative, car les six citations des discours de Pierre, ci-haut 
mentionnées, sont aussi à portée christologique. On pourrait facilement multiplier les exemples, mais ceux qui sont indiqués ici suffisent à illustrer notre propos.

En outre, cet Ancien Testament est cité aussi bien à partir du texte hébraïque que de la version grecque, la Septante (LXX), voire même de la recension de Théodotion. Par exemple, la mention de *vierge " en Mt 1,23 provient d'Is 7,14 LXX, tandis que le texte hébraïque parle de *jeune femme. 1 Cor 15,54 cite Is 25,8 sous une forme qui ne correspond ni à la LXX ni à Aquila ni à Symmaque, mais seulement et exactement à Théodotion.

Le Nouveau Testament ne se limite pas à citer l'Ancien. En effet, on retrouve aussi des citations empruntées à la littérature grecque et à la littérature juive. Ainsi, au dire de Clément d'Alexandrie, Ti 1,12 cite Épiminide de Cnosos, poète crétois du VI' siècle av. J.-C.; Ac 17,28 cite le philosophe Aratus; 1 Cor 15,33 cite le poète comique Ménandre, dans Thaïs; etc. Du côté de la littérature juive, on peut signaler Jude 14 qui cite Hénoch 1,9; la mention de Jannès et Jambrès en $2 \mathrm{Tm} 3,8$ qui se retrouve dans le Targum d'Ex 1,15 et 7,11 dont l'antiquité est confirmée par le Document de Damas au chapitre 5,18-19; Mc 13,22 qui cite Dt 13,2 , non selon le texte hébraïque ni selon la traduction grecque, mais bien selon le Targum comme le montre l'emploi du pluriel et la mention du faux prophète; Eph 4,8 qui cite le Targum du Ps 68,19 ; Jn 11,49b-50 qui cite le grand prêtre Caïphe; etc.

Dans tous ces cas, que la citation provienne du texte hébraïque, de la paraphrase araméenne du Targum ou de différentes traductions grecques, il ne faut pas oublier de souligner que le rapport de l'ensemble du Nouveau Testament à ces textes se définit d'abord en termes d'achèvement, d'accomplissement, de plénitude et de perfection. C'est à partir du Christ que ces citations et allusions reçoivent la plénitude de leur sens. Un dernier exemple suffira à illustrer et éclairer cette affirmation. Dans Gal 3,11, Paul cite Hab 2,4, mais comme c'est sa christologic qui devient le principe de sa lecture, il le fait avec une liberté audacieuse. Le texte hébraïque d'Hab 2,4 dit: - le juste vivra par sa fidélité *, la LXX traduit: :Le juste vivra par la fidélité de Dieu ", et Paul écrit: - le juste vivra par la foi ". Ce ne sont donc plus les citations ou allusions tirées des textes de la tradition juive qui sont les principales références, mais plutôt Jésus-Christ. Ce sont certes les textes porteurs qui commentent ces citations et allusions, mais c'est l'événement Jésus-Christ * qui en est la clé d'interprétation. 
Ces quelques lignes, trop brèves, sur l'intertextualité biblique illustrent néanmoins, de façon suggestive, l'unité entre l'Ancien Testament et le Nouveau Testament. Dans cette perspective, il ne serait pas exagéré de dire que les 46 volumes de l'Ancien Testament et les 27 du Nouveau Testament forment une seule œuvre. Cette idée est d'ailleurs matérialisée par le fait que cette véritable bibliothèque, dont les livres parcourent une histoire bimillénaire, est regroupée en un seul livre: la Bible.

\section{Intertextualité et liturgie}

La Guézéra shawa est une des treize règles d'interprétation élaborées au deuxième siècle de notre ère par Rabbi Ishmaël. Un dictionnaire usuel hébreu-français traduit cette expression par * analogie sémantique ". La Guézéra shawa consiste, pour reprendre une belle formule d'Emmanuel Lévinas, à associer un "paysage" biblique à un autre pour dégager de ce jumelage le parfum secret du premier.12. À la lumière de cette définition, on pourrait traduire ce terme par *intertextualitén. En effet, celui-ci exprime très bien ce qui est en jeu dans cette manière d'approcher le(s) texte(s).

Mais cette pratique de l'intertextualité (Guézéra shawa) date bien avant Rabbi Ishmaël. Ce jeu de l'intertextualité remonte déjà, nous l'avons vu, à l'Ancien Testament et au Nouveau Testament, mais il remonte aussi aux vieilles pratiques des lectures synagogales. Par exemple, dans les synagogues de Palestine, on sait que le texte des Prophètes était choisi de manière à faire résonner le message de Moïse; une affinité thématique et des mots en commun devaient donc relier ces deux textes. Puis, dans son commentaire, l'homéliste devait utiliser des versets tirés principalement des Écrits de sagesse. À nouveau, ce texte sapiential devait être relié au texte du Pentateuque par un accrochage verbal, un mot commun ou un thème similaire. Ainsi était réalisée l'union des trois grandes parties de l'Ancien Testament: Torah, Prophètes et Écrits sapientiaux. Ces textes, lus et commentés en quelque sorte en miroir, mettaient en valeur le message de la Torah à travers le prisme des autres textes bibliques. Ce libre jeu d'intertextualité s'attachait à rendre vivante la Torah, à l'actualiser en fonction des problèmes concrets de la communauté.

12 Quatre lectures talmudiques, Ed. de Minuit, 1968, p. 120. 
58

Or, n'oublions pas que les premiers chrétiens continuèrent de fréquenter la synagogue, à l'exemple de leur maître Jésus. Il n'est pas étonnant alors de constater que les lectures de la liturgie chrétienne ne sont qu'un développement de celles de la synagogue. C'est pourquoi on retrouve dans nos liturgies le même phénomène d'intertextualité biblique, c'est-à-dire le même phénomène d'aimantation textuelle, verbale et thématique, mais cette fois-ci principalement avec les textes du Nouveau Testament qui sont lus et commentés à la suite et à la lumière des textes de l'Ancien Testament. C'est aussi pourquoi, aujourd'hui comme il y a plus de 2000 ans dans les synagogues de Palestine, c'est encore le même souci de rendre vivants les textes qui préoccupe le responsable de la liturgie chrétienne.

\section{Postlude}

La Bible est une véritable bibliothèque. Or, la simple juxtaposition des livres dans une bibliothèque est, du point de vue strictement spatial, l'expression visible du phénomène d'intertextualité. Mais ce bref exposé a bien montré que l'intertextualité biblique ne se joue pas seulement au niveau des livres; elle se joue dans les phrases et dans les mots; et tout particulièrement dans les citations et les allusions qui sont toujours laissées à la sagacité et la perspicacité du lecteur. Pour un lecteur averti, lire un texte biblique, c'est toujours lire beaucoup plus qu'un texte biblique: bien entendu, c'est relire sa propre mémoire littéraire, mais aussi, à travers elle, c'est tenter de relire celle du texte même et de ce fait tout texte biblique est un palimpseste. En effet, comprendre un texte biblique n'est jamais rien d'autre que de le mettre en relation avec des contextes différents: celui des autres textes de l'œuvre même, des autres textes de la Bible, voire même, comme le montre notre propre article, des textes de son temps et enfin de la littérature prise comme un tout.

Finalement, la dernière partie de notre exposé a bien montré que le jeu de l'intertextualité biblique n'a jamais cessé. Commencée dans l'Ancien Testament, relayée par les synagogues, poursuivie par le Nouveau Testament, l'intertextualité biblique se continue encore de nos jours dans chacune de nos assemblées dominicales. Aujourd'hui comme hier, l'intertextualité biblique est le lieu où se vérifie la constante actualité de l'Écriture. 